\title{
Assessing Lipoprotein Levels in Normotensive and Hypertensive Pregnant Women in Port Harcourt, Nigeria
}

\author{
*Oladapo-Akinfolarin, Tomaziga Tomiloba; Bartimeaus, Ebirien-Agana Samuel; Nwachuku, \\ Edna.Ogechi and Nduka, Nsirim
}

Department of Medical Laboratory Science, Rivers State University, PM.B. 5080, Nkpolu-Oroworukwo, Port Harcourt, Nigeria.

\section{*Corresponding Author}

Oladapo-Akinfolarin, Tomaziga Tomiloba

Department of Medical Laboratory Science

Rivers State University

PM.B. 5080, Nkpolu - Oroworukwu

Port Harcourt

Nigeria

Email: ola_atomaziga@yahoo.com

Received:15 December 2017 ; | Revised:28 December 2017; | Accepted: 12 March 2018

\section{Abstract}

Background: Lipids and lipoproteins are central to the energy metabolism of the liver, and have continued to be important in clinical practice; basically because of their association with Coronary Heart Disease (CHD). Abnormal lipid metabolism is linked to atherosclerosis due to rising incidence of abdominal obesity.

Objective: To evaluate some cardiovascular disease risk status such as lipid profile of pregnant Normotensive and Hypertensive women and compare it with the corresponding levels in Non pregnant Normotensive women.

Method: This study was conducted in the Department of Medical Laboratory Science, Rivers State University, Port Harcourt, Nigeria .A total of 300 women were registered for the study after taking informed consent. 100 of them were pregnant hypertensives, 100 pregnant Normotensives and 100 Non pregnant normotensives as control. All relevant information was recorded on a predesigned questionnaire. Serum total cholesterol (TC), High-density lipoprotein (HDL-C), low density lipoprotein cholesterol (LDL-C), very lowdensity lipoprotein cholesterol(VLDL-C) and Triglyceride levels were measured in the 200 women, including systolic and diastolic blood pressure (SBP \& DBP) and BMI; and compared with that of the 100 non pregnant Normotensive women.

Result: Total Cholesterol levels were not statistically significant between the subject groups and control. Mean values of TG, HDL-C, LDL-C and VLDL-C were significant at $\mathrm{p}<0.05$. Age, BMI, SBP and DBP were also significant at $\mathrm{p}<0.05$. BMI, SBP and DBP, TG, HDL and VLDL were statistically significant when HPW were compared with NPW. Age, BMI, SBP and DBP, TG, HDL-C and LDL-C were statistically significant when HPW were compared with NNPW. Only, BMI and TG were statistically significant at 19 $\mathrm{P}<0.05$ when NPW were compared with NNPW. $90 \%$ of HPW at $38.72 \pm 6.56,14 \%$ of NPW at $36.16 \pm 12.74$, and $2 \%$ of NNPW at $31.38 \pm 0.88$ were obese. HPW and NPW had higher ratios of TG: HDL (75\% and $74 \%)$ 
compared to NNPW of $10 \%$. NPW and NNPW had higher ratios of TC: HDL (65\% and 48\%) compared to HPW of 4\%. NPW and HPW had higher ratio of LDL: HDL (17\% and 14\%) compared to NNPW of $13 \%$.

Conclusion: Lipoprotein levels and ratios are higher in hypertensive and Normotensive pregnant women, than in Normotensive Non pregnant women; thereby, exposing them to cardiovascular risks in the near future.

Keywords: Hypertensive, Normotensive, Pregnant women

\section{Introduction}

WHO predicts 11.1 million deaths globally as well as $71 \%$ deaths in developing countries of the world due to Coronary Artery Disease (CAD) by the year $2020^{[1]}$. Several risk factors have been found to be associated with CAD such as, Age, sex, and Hypertension ${ }^{[2]}$.Lipids and lipoproteins are central to the energy metabolism of the liver, and have continued to be important in clinical practice, basically because of their association with coronary Heart Disease (CHD) ${ }^{[3]}$. Studies have demonstrated that in countries where high fat consumption is high like Port Harcourt where carbohydrate and fat are our stable food; there is a clear association between the blood lipid levels and the development of atherosclerosis due to rising incidence of abdominal obesity ${ }^{[2]}$.

Dyslipidaemia has been identified as one of the most important risk factors associated with CAD by the INTER-SOUTH ASIA STUDY ${ }^{[1]}$. Over the years, studies have added value to knowledge concerning the nature of lipoproteins and their lipid and protein parts, as well as their role in the pathogenesis of the atherosclerotic process [3] During pregnancy, maternal metabolism must satisfy the demands of the developing fetus as well the energy requirements of the mother. In early pregnancy, there is usually an increased hepatic production of triglyceride and facilitated removal of triglycerides from the circulation, resulting in an increased deposition of fat in maternal adipose tissue $^{[1]}$. In late pregnancy, however, there is an enhanced release of fatty acid from adipocytes as a result of resistance by insulin and stimulation of hormone-sensitive lipase by placental hormone. These metabolic changes result in the metabolism of the gravid female to store energy in pregnancy in order to cope with the energy requirements of late gestation ${ }^{[4]}$. The outcome is the specific alteration of the maternal lipid metabolism during pregnancy. Studies have shown that Total Cholesterol and phospholipids are moderately increased while TG is highly increased ${ }^{[5]}$. Low HDL-C, High TG and high LDL-C levels have also been associated with increased incidence of CAD ${ }^{[6]}$. High amounts of triglycerides are also found in the very low density lipoprotein and High density lipoprotein fractions during late pregnancy ${ }^{[7]}$. The mechanism of action for this alteration is in two phases. Firstly, increased levels of estrogen during pregnancy lead to an increased hepatic synthesis of VLDL highly rich in Triglyceride ${ }^{[8]}$. Secondly, lipoprotein triglyceride are removed slowly as a result of impaired or reduced capacity of lipoprotein lipase(LPL) and Hepatic triglyceride Lipase (HL), with HL being mostly affected ${ }^{[9]}$. The surplus VLDL triglycerides causes a further push of triglycerides to lipoproteins of higher density by the cholesteryl ester transfer protein (CETP) ${ }^{[10]}$; thereby, shifting HDL subclass toward larger, triglyceride-rich species in late gestation $^{[11]}$. During pregnancy, LDL particles get buoyant in TG too; but, to some extent become smaller and denser compared to HDL [12]. Researchers have shown that two patterns of LDL subclass distribution $A$ and $B$ could be differentiated using non denaturing gradient gel electrophoresis. Austin and his collegues in 1988 showed in their demonstration that Pattern A had predominant LDL particles that are large and buoyant, while pattern B had predominantly small, dense LDL particles ${ }^{[13]}$. The larger more buoyant subclass of LDL is predominantly in healthy females of reproductive age, while smaller, denser LDL occurs after menopause ${ }^{[1]}$. The small dense LDL particles are usually more susceptible to oxidation, and show stronger adhesion to proteoglycans of the vessel wall, thereby exhibiting a reduction in the uptake by the LDL receptor ${ }^{[14]}$. Plasma triglycerides account for about $40-60 \%$ of 
the diversity in small, dense LDL concentrations in men and women who are not pregnant ${ }^{[15]}$. Studies have shown that, there is a correlation between increased levels of plasma triglyceride, small dense LDL $^{[16]}$, and decreased HDL cholesterol levels [12], a metabolic situation termed atherogenic lipoprotein phenotype ${ }^{[1]}$. Elevated triglycerides and the accumulation of small dense LDL during pregnancy are thought to increase the risk for endothelial damage ${ }^{[12]}$, and thus Cardiovascular Disease. This work is aimed at assessing lipoprotein levels in Normotensive and Hypertensive pregnant women in Port Harcourt, Nigeria.

\section{Materials and Methods}

The study was conducted in the Department of Medical Laboratory Science, Rivers State University, Nkpolu Oroworukwo, Port Harcourt. 300 women were enrolled in our study after informed consent. The study subjects were divided into three groups. 100-pregnant hypertensive women(HPW), 100- Normotensive pregnant women (NPW) attending antenatal care at Braithwaite Memorial Specialist Hospital (BMSH), Port Harcourt, and 100 Normotensive non pregnant Women (NNPW) as control. The control group comprised healthy non pregnant women between 18 and 30 years. All subjects were non-smokers, non alcoholics and there was no positive family history of CAD. Hypertension was defined as BP $\geq$ 140/90mmHg on several occasions. Anthropometric assessment was done which included, Height (m), Weight $(\mathrm{kg})$, and calculation of Body Mass Index $(\mathrm{BMI})=$ Weight $(\mathrm{kg}) /$ Height $(\mathrm{m} 2)$. A detailed history, clinical examination was recorded for all study subjects. $2 \mathrm{ml}$ of peripheral venous blood was collected from anticubital vein using a dry, disposable syringe under sterile conditions, in a sterile plain bottle. Serum was separated by centrifugation at 3000rpm, for 15minutes. Serum was stored in refrigerator till ready for use; and used for estimation of total cholesterol, HDL-C, TG, and VLDL-C. The tests were carried out in a semiautomated clinical chemistry auto analyzer (MAPLAP PLUS) using standard reagent kits
(ATLAS). The following tests were done as part of routine lipid profile: Serum Total cholesterol (TC), Triglyceride (TG), HDL cholesterol (HDL-C), Very low density lipoprotein (VLDL-C) and LDL cholesterol (LDL-C) calculated using Freidwald formula. The atherogenic ratios were calculated as follows: Atherogenic Index of plasma (AIP) $=\log$ (TG/HDL-C)

\section{Castelli Risk Index (CRI-1) $=$ TC $/$ HDL-C Castelli Risk Index (CRI-2) = LDL-C / HDL-C.}

Statistical analyses were performed with the IBM SPSS version 20.0 software. Comparism of variables, mean and standard deviation, ANOVA Post Hoc and Pearson's coefficient of determination were used.

\section{Results}

Anthropometric and biochemical indices among the study groups are described in Table 1; Age, BMI, Systolic and Diastolic blood pressures were significantly high in HPW and NPW compared to the control (NNPW) at $\mathrm{p}<0.05$. TG, HDL-C, LDL-C were significant in HPW and NPW at $\mathrm{p}<0.05$ compared to NNPW. TC was not statistically different between the case groups and control.

Table 2 reflects the results of Anthropometric and biomedical indices using post-Hoc findings within the study groups. This table shows biochemical predictions between two different groups indicating significant association. Comparing $\mathrm{p}$ values for NNPW vs NPW; BMI and TG were significant at $\mathrm{p}<0.05$. TC, HDL-C, and LDL-C and other anthropometric parameters including age, were not significant. Comparing the values for NNPW vs HPW; TG, HDL-C, LDL-C and other anthropometric parameters(Age, BMI, SBP and DBP) were significant at $\mathrm{p}<0.05$. TC was not significant. Comparing the anthropometric and biochemical parameters for NPW vs HPW; All including age were significant at $\mathrm{p}<0.05$ except $\mathrm{TC}$. 
Table 1:Anthropometric and Biochemical indices among the study groups

\begin{tabular}{|c|c|c|c|c|c|}
\hline Parameter & NNPW $(\mathrm{n}=100)$ & NPW(n=100) & HPW $(\mathrm{n}=100)$ & Fvalue & Pvalue \\
\hline Age $(\mathrm{yrs})$ & $28.68 \pm 6.60$ & $30.25 \pm 9.74$ & $32.70 \pm 5.30$ & 11.93 & $<0.001$ \\
\hline BMI $\left(\mathrm{Kg} / \mathrm{m}^{2}\right)$ & $23.74 \pm 2.85$ & $27.36 \pm 1.46$ & $37.59 \pm 7.10$ & 136.44 & $<0.001$ \\
\hline $\mathrm{SBP}(\mathrm{mm} / \mathrm{Hg})$ & $100.80 \pm 3.94$ & $104.10 \pm 5.48$ & $153.40 \pm 19.24$ & 306.4 & $<0.001$ \\
\hline $\mathrm{DBP}(\mathrm{mm} / \mathrm{Hg})$ & $70.20 \pm 1.41$ & $66.80 \pm 6.20$ & $96.10 \pm 14.35$ & 194.701 & $<0.001$ \\
\hline $\mathrm{TC}(\mathrm{mmol} / \mathrm{l})$ & $4.99 \pm 1.19$ & $5.19 \pm 0.79$ & $5.43 \pm 1.75$ & 2.24 & 0.108 \\
\hline $\mathrm{TG}(\mathrm{mmol} / \mathrm{L})$ & $0.89 \pm 0.42$ & $1.92 \pm 0.71$ & $2.46 \pm 0.80$ & 132.63 & $<0.001$ \\
\hline $\mathrm{HDL}-\mathrm{C}(\mathrm{mmol} / \mathrm{L})$ & $1.76 \pm 0.56$ & $1.58 \pm 1.34$ & $2.27 \pm 1.22$ & 13.35 & $<0.001$ \\
\hline $\mathrm{LDL}-\mathrm{C}(\mathrm{mmol} / \mathrm{L})$ & $2.78 \pm 1.25$ & $2.74 \pm 4.16$ & $2.10 \pm 1.71$ & 6.4 & 0.001 \\
\hline VLDL-C(mmol/l) & $0.18 \pm 0.08$ & $0.38 \pm 0.16$ & $0.49 \pm 0.16$ & 37.45 & $<0.001$ \\
\hline & & & & & \\
\hline
\end{tabular}

NNPW_-Normotensive Non-Pregnant Women, NPW_-Normotensive Pregnant Women, HPW-Hypertensive Pregnant Women, TC-Total cholesterol, TG-Triglyceride, HDL-High density lipoprotein, LDL-Low density lipoprotein, VLDL-Very low density lipoprotein.

Table 2:The ANOVA Post-Hoc findings within the study group

\begin{tabular}{|c|c|c|c|c|c|c|}
\hline Parameters & $\begin{array}{c}\text { NNPW } \\
\text { vs NPW } \\
\text { (P. Value) }\end{array}$ & $\begin{array}{c}\text { NNPW } \\
\text { vs NPW } \\
\text { (q. value) }\end{array}$ & $\begin{array}{c}\text { NNPW } \\
\text { VS } \\
\text { HPW(P-value) }\end{array}$ & $\begin{array}{c}\text { NNPW } \\
\text { VS } \\
\text { HPW(q-value) }\end{array}$ & $\begin{array}{c}\text { NPW } \\
\text { VS } \\
\text { HPW(P-Value) }\end{array}$ & $\begin{array}{c}\text { NPW } \\
\text { VS } \\
\text { HPW(q-value) }\end{array}$ \\
\hline Age & 0.136 & 0.864 & $<0.001$ & 1.000 & 0.010 & 0.990 \\
\hline BMI & $<0.001$ & 1.000 & $<0.001$ & 1.000 & $<0.001$ & 1.000 \\
\hline SBP & 0.311 & 0.689 & $<0.001$ & 1.000 & $<0.001$ & 1.000 \\
\hline DBP & 0.1 & 0.900 & $<0.001$ & 1.000 & $<0.001$ & 1.000 \\
\hline TC & 0.832 & 0.168 & 0.102 & 0.898 & 0.305 & 0.695 \\
\hline TG & $<0.001$ & 1.000 & $<0.001$ & 1.000 & $<0.001$ & 1.000 \\
\hline HDL-C & 0.335 & 0.665 & 0.001 & 0.999 & 0.001 & 0.999 \\
\hline LDL-C & 0.853 & 0.147 & 0.003 & 0.997 & 0.015 & 0.985 \\
\hline VLDL-C & $<0.001$ & 1.000 & $<0.001$ & 1.000 & $<0.001$ & 1.000 \\
\hline
\end{tabular}

BMI-Body mass index, Tc- Total cholesterol, TG-Triglyceride,

HDL-High density lipoprotein, LDL-Low density lipoprotein ,

VLDL- Very low density lipoprotein. 
Table 3: Risk profile of subjects using AIP

\begin{tabular}{|c|c|c|c|}
\hline Subjects & $\begin{array}{c}\text { Low risk } \\
-0.3-0.1\end{array}$ & $\begin{array}{c}\text { Moderate risk } \\
0.1-0.24\end{array}$ & $\begin{array}{c}\text { High risk } \\
>0.24\end{array}$ \\
\hline NNPW & $-0.09 \pm 0.11(90 \%)$ & $0.16 \pm 0.04(10 \%)$ & $0.49 \pm 0.32(28 \%)$ \\
\hline NPW & $0.04 \pm 0.05(26 \%)$ & $0.25 \pm 0.05(46 \%)$ & $0.39 \pm 0.18(30 \%)$ \\
\hline HPW & $0.06 \pm 0.03(25 \%)$ & $0.19 \pm 0.04(45 \%)$ & \\
\hline
\end{tabular}

AIP_-Atherogenic Index of Plasma

Table 4:Risk profile of subjects using CRI 1

\begin{tabular}{|c|c|c|c|c|}
\hline & \multicolumn{2}{|c|}{$\begin{array}{c}\text { Low risk } \\
(<1-3)\end{array}$} & \multicolumn{2}{c|}{$\begin{array}{c}\text { Moderate risk } \\
(3-5)\end{array}$} \\
\hline NNPW & $2.22 \pm 0.42 \quad(52 \%)$ & $3.67 \pm 0.55$ & $(44 \%)$ & $6.47 \pm 0.56$ \\
\hline NPW & $2.19 \pm 0.57 \quad(35 \%)$ & $3.87 \pm 0.51 \quad(51 \%)$ & $9.28 \pm 5.39 \quad(14 \%)$ \\
\hline HPW & $1.31 \pm 0.62 \quad(86 \%)$ & $3.5 \pm 0.91$ & $(2 \%)$ & $6.24 \pm 0.22 \quad(2 \%)$ \\
\hline
\end{tabular}

\section{CRI1 - Castelli Risk Index 1}

Table 5: Risk Profile using CRI(II)

\begin{tabular}{|c|c|c|c|}
\hline & $\begin{array}{c}\text { Low risk } \\
(<1-3)\end{array}$ & $\begin{array}{c}\text { Moderate risk } \\
(3-5)\end{array}$ & $\begin{array}{c}\text { High risk } \\
(>5)\end{array}$ \\
\hline NNPW & $1.45 \pm 0.66(87 \%)$ & $3.51 \pm 0.26 \quad(10 \%)$ & $5.61 \pm 0.16 \quad(3 \%)$ \\
\hline NPW & $1.58 \pm 0.82(83 \%)$ & $3.55 \pm 0.27 \quad(9 \%)$ & $10.07 \pm 0.63(8 \%)$ \\
\hline HPW & $0.95 \pm 0.79(86 \%)$ & $4.03 \pm 0.69 \quad(7 \%)$ & $6.97 \pm 0.29 \quad(7 \%)$ \\
\hline
\end{tabular}

CRI(II)—Castelli Risk Index II 
Table 6: Risk profile of subjects using BMI

\begin{tabular}{|l|c|c|c|c|}
\hline & DEFICIENT & NORMAL & OVER WEIGHT & OBESE \\
\cline { 2 - 5 } & $<18.5$ & $18.5-24.5$ & $>24.5-29.5$ & $>29.5$ \\
\hline NNPW & $17.20 \pm 1.23(2 \%)$ & $22.33 \pm 1.45(65 \%)$ & $26.78 \pm 1.40(31 \%)$ & $31.38 \pm 0.88(2 \%)$ \\
\hline & - & $23.20 \pm 0.98(28 \%)$ & $27.24 \pm 1.34(58 \%)$ & $36.16 \pm 12.74(14 \%)$ \\
\hline NPW & - & - & $27.41 \pm 1.14 \quad(10 \%)$ & $38.72 \pm 6.56(90 \%)$ \\
\hline HPW & - & & & \\
\hline
\end{tabular}

BMI—Body Mass Index

Table 3 reflects the risk profile of subjects across the 3 groups using Atherogenic Index of Plasma (AIP). $75 \%$ of HPW were at moderate and high risk $(45 \%, 30 \%) ; 74 \%$ NPW were at moderate and high risk $(46 \%, 28 \%)$ and $10 \%$ of NNPW at moderate risk. For low risk, NNPW (90\%), NPW (26\%) and HPW (25\%) were recorded.

Table 4 reflects the risk profile of subjects across the 3 groups using Castelli Risk Index 1. The highest percentage for high risk was in NPW (14\%); NNPW and HPW had (4\% and 2\%) respectively. For moderate risk NPW (51\%), NNPW and HPW $(44 \%, 2 \%)$ respectively. The highest percentage for low risk was in $\operatorname{HPW}(86 \%)$; NNPW and NPW had (52\% and $35 \%)$ respectively.

Table 5 reflects the risk profile of subjects across the 3 groups using Castelli Risk Index 2 . The highest percentage of high risk was in NPW(8\%), HPW and NNPW had (7\% and 3\%) respectiely. For moderate risk NNPW(10\%), NPW and HPW (9\% and 7\%) respectively. The highest percentage for low risk was in NNPW (87\%), HPW and NPW $(86 \%, 83 \%)$ respectively.

Table 6 reflects the risk profile of subjects using BMI. With the classification as deficient, normal, overweight and obese. 2\% were for NNPW, (65\% and 28\%) for NNPW and NPW, 31\%, 58\% and $10 \%$ for NNPW, NPW and HPW, and (2\%, $14 \%$ and $90 \%$ ) for NNPW, NPW and HPW respectively.

\section{Discussion}

Lipid profile refers to some routinely done biochemical tests to assess the atherogenic status of individuals at risk of Coronary Artery Disease (CAD). It includes serum Triglycerides (TG), Total cholesterol (TC) and the sub fractions LDL-C and HDL-C. Cardiovascular disease has been, and remains a threat to women especially those of child bearing age prone to some cardiovascular risk factors. It is the leading cause of death worldwide involving $80 \%$ in low and medium income countries ${ }^{[17]}$. Researchers over the years have tried to find a connotation between lipids and hypertension in pregnant states, in order to reduce mortality and morbidity in women of child bearing age. Kamel and his colleagues in 2014 found out that risk factors like diabetes in pregnancy could cause increased risk for vascular disease in the offspring due to disturbed lipid profile ${ }^{[28]}$, although their work was on rats. Because of altered lipid metabolism during pregnancy, total cholesterol and phospholipids are moderately increased while TG is highly increased according to Winkler et al., (2000) [1]. Our finding in this study was similar, but TG was highly significantly increased in HPW compared to NPW and NNPW $(2.46 \pm 0.80,1.92 \pm$ $0.71,0.89 \pm 0.42)$. TC was moderately increased but not significantly increased statistically $(5.43 \pm 1.75$, 
$5.19 \pm 0.79$, and $4.99 \pm 1.19)$. However, this work agrees with Jan and his friends in 2012, who found out in their research work that TC was not statistically significant while assessing lipoproteins in normal and pregnancy induced hypertensive pregnant women in Tertiary care Hospitals ${ }^{[18]}$. Previous studies showed that TG is markedly increased causing a dramatic damage in the lipid and lipoprotein profile in normal pregnancy compared to non pregnant women. The observation holds true to an extent in this study. Here the serum TG concentrations showed very significant increases $(\mathrm{p}<0.05)$ in HPW and NPW compared to NNPW. The mean value being raised above 2 folds in HPW compared to NNPW. HDL-C, LDL-C and VLDL-C also increased significantly $(\mathrm{p}<0.001)$.This agrees partly with Neboh et al., (2012) and Udoh, et al., (2002) ${ }^{[19,20]}$, who observed a progressive increase in TG, HDL-C, LDL-C, VLDL-C and TC at various stages of pregnancy; even if we did not specify the stages of pregnancy in this work, but in contrast to their finding, TC was not significant. The mean increase in TG levels in HPW and NPW are higher than that of NNPW because of estrogen levels in pregnancy. CAD has been associated with alterations in lipid metabolism, which include hypertriglyceridemia and significantly reduced HDL with reduced HDL being an independent risk factor for increased exposure to cardiovascular disease ${ }^{[1]}$. The pattern observed in this study is very characteristic of dyslipidemia with obesity as a transient factor. TG, LDL-C and HDL$\mathrm{C}$ and VLDL-C were significantly increased. According to Bhardwaj et al.,(2013), obesity causes a rise in small dense LDL particles which are not measured routinely ${ }^{[2]}$. Atherogenic lipoprotein phenotype is characterized by high TG, low HDL and rise in small dense LDL. In such a case, going beyond the routinely done lipid profile becomes essential. The cardiac risk factor (TC/HDL) was calculated for the various groups, as a predictor of atherosclerosis in HPW and NPW. A greater increase was observed in NPW, which disagrees with the findings of Neboh et al., (2012) and De et al., (2006) who reported a decrease in TC/HDL during pregnancy ${ }^{[19,21]}$.

In this work, the greater percentage for high risk was recorded in NPW compared with HPW and NNPW. The highest percentage for low risk was recorded in HPW. In addition to the relevance of TC/HDL as a predictor of atherosclerosis, the significance of altered $\mathrm{TC} / \mathrm{HDL}$ ratio is an indication of additional risk in pregnancy. The cardiac risk factor (LDL/HDL) was also calculated for the three groups, as a predictor of atherosclerosis in HPW and NPW. A greater increase was observed in NPW for moderate and high risk (17\%), compared to (14\% in HPW) and $13 \%$ in NNPW). NPW also recorded the least score for the low risk compared to the other two groups, implying the significance of altered LDL/HDL ratio as an indication of additional risk in pregnancy. The cardiac risk factor (logTG/HDL) was assessed among the three groups. HPW had $75 \%$ for moderate and high risk, followed by NPW (74\%) for moderate and high risk compared with the control that had $10 \%$ for moderate alone. This study shows a strong correlation between AIP and BMI compared to other cardiac risk factors used. The HPW were $90 \%$ obese and $10 \%$ over weight. This agrees with Niroumand et a.,(2015) that Women with higher BMI have a higher AIP too and with findings of previous studies that show strong association between abnormalities of blood lipoprotein and different habits of people, such as life style and eating habits ${ }^{[22]}$. Flier.(2008) also demonstrated that there is a correlation between increased BMI and significant increase in values of HDL-C, LDL-C, and TG, as well as systolic blood pressure and diastolic blood pressure ${ }^{[23]}$. Our finding is similar to those of Niroumond et al.,(2015) and Kearns et al., (2014), who found out that, chronic diseases like hypertension, diabetes, dyslipidemia and osteoarthritis are more common in obese persons ${ }^{[22,24]}$

According to Flier.(2008), regular physical activity can help to control BMI, improve lipid profile and decrease the risk of coronary heart disease, heart attack, high BP and diabetes ${ }^{[23]}$. This study also demonstrated a direct relationship between AIP and the level of physical activity. This association was higher in NPW and HPW than NNPW. This could imply that obesity is a transient factor based on the state of pregnancy especially when accompanied with hypertension. According to the values of AIP previously mentioned; this study, showed that NPW and HPW had higher percentages for higher risk, therefore were at a higher risk of 
developing coronary heart disease. This agrees with Niroumond et al.,(2015) who found higher AIP values to expose subjects at higher risk for developing coronary heart disease ${ }^{[22]}$. Various studies have illustrated a strong correlation between AIP and lipoproteins particle size, considering it to be an indicator of atherogenic lipoprotein status ${ }^{[25]}$. As was observed in this study, significant increase in AIP was detected with increasing TG, LDL-C, and HDL-C.

Studies have shown that AIP plays a predictive value for atherosclerosis ${ }^{[26]}$, and could be used as an index of highest sensitivity for assessing cardiovascular risks, and also used for predicting acute coronary events ${ }^{[25]}$. More so, in situations where all parameters are normal, AIP may be the alternative tool ${ }^{[27]}$. As was the case in this study; $75 \%$ of HPW were at moderate and high risk, $74 \%$ of NPW were at moderate and high risk even if TC was not significantly different, and HDL-C was high in HPW compared to the control, disagreeing partly with Neboh et al., (2012) who showed that normal pregnancy may not put women at risk ${ }^{[19]}$. This work agrees with their study that corresponding increase in HDL-C alongside other lipid fractions may reduce cardiac risk factor, signifying that pregnant women are in fact protected from the risk of atherosclerosis more than non pregnant women as gestational age increases; Though gestational age was not particularly considered in phases in this research, AIP in addition to other cardiac risk factors proves otherwise.

\section{Conclusion}

Our findings showed that lipoprotein levels and ratios are higher in hypertensive and Normotensive pregnant women, compared to normotensive non pregnant women and therefore, exposes them to cardiovascular risks in the near future if not controlled.

\section{Recommendation}

Based on the present study, Healthy diet, regular exercise and modest life style are recommended for women, and the two Cardiac risk factors should be done with AIP as lipid ratios in assessing for cardiac risks.

\section{Aknowledgement}

We sincerely appreciate the contributions of Dr. Boisa, N., Mr Akinfolarin, O.M. and Mr Obisike, U.

\section{References}

1 Winkler, K., Wetzka, B., Hoffmann, M.M., Friedrich,I., Kinner,M., Baumstark, M.W., Wieland, H., Marz, W., Zahradnik, H.P. Low density lipoprotein (LDL) subfractions during pregnancy: accumulation of buoyant LDL with advancing gestation. J Clin Endocrinol Metab 2000; 85(12): 4543-4550 [PMID: 11134106 DOI: $10.1210 /$ jcem.85.12.7027]

2 Bhardwaj, S., Bhattacharjee, J., Bhatnagar, M.K;Tyagi, S. Atherogenic Index of Plasma, Castelli Risk Index and Atherogenic Coefficient- New Parameters in Assessing Cardiovascular Risk, International Journal of Pharmacy and Biological Sciences,2013,3(3),359-364.

3 Bishop, M.L. Lipids and Lipoproteins, Techniques, principles and Correlations In clinical chemistry, Fody, E.P.; Schoeff, L.E. 6th Edn, Lippincott. Williams \& Wilkins. Philadelphia, 2010, pp 328-351.

4 Stock, M.J. and Metcalfe , J. Maternal physiology during gestation. In: Knobil, E. and Neill, JD, Eds. The physiology of Reproduction. New York: Raven Press: 1994, pp 947-983.

5 Peters, J. P., Heinemann, M., Man, E. B. The lipids of serum in pregnancy. Journal of Clinical Investigation, 1957, 30, 388-394.

6 Weissglas-volkor, D., Pajukanta, P. Genetic causes of High and Low Serum HDL cholesterol, The Journal of Lipid Research, 2010,51,2032-2057.

7 Knopp, R.H. Bonet, M. A., Lasuncion, A., Montelongo, A.H. Lipoprotein metabolism in pregnancy, In: Herrera, E., Knopp, R.H. 
editions. Perinatal Biochemistry. Boca Raton: CRC press, 1992, pp19-51.

8 Walsh BW, Schiff I, Rosner B, Greenberg L, Ravnikar V, Sacks FM. Effects of postmenopausal estrogen replacement on the concentrations and metabolism of plasma lipoproteins. N Engl J Med 1991; 325(17): 1196-1204 [PMID: 1922206 DOI: 10.1056/NEJM199110243251702]

9 Sattar, N., Greer, I.A., louden, J. et al. Lipoprotein sub-fractions changes in normal pregnancy: threshold effect of plasma triglyceride in appearance of small dense low density lipoprotein. Journal of clinical Endocrinology and metabolism, 1997, 82, 2483-2491.

10 Silliman K, Tall AR, Kretchmer N, Forte TM. Unusual high-density lipoprotein subclass distribution during late pregnancy. Metabolism 1993; 42(12): 1592-1599 [PMID: 8246775]

11 Alvarez JJ, Montelongo A, Iglesias A, Lasuncion MA, Herrera E. Longitudinal study on lipoprotein profile, high density lipoprotein subclass, and postheparin lipases during gestation in women. J Lipid Res 1996; 37(2): 299-308 [PMID: 9026528]

12 Hubel CA, Shakir Y, Gallaher MJ, McLaughlin MK, Roberts JM. Low-density lipoprotein particle size decreases during normal pregnancy in association with triglyceride increases. J Soc Gynecol Investig 1998; 5(5): 244-250 [PMID: 9773399]

13 Austin MA, Hokanson JE, Brunzell JD. Characterization of low-density lipoprotein subclasses: methodologic approaches and clinical relevance. Curr Opin Lipidol 1994; 5(6): 395-403 [PMID: 7712044]

14 Anber V, Griffin BA, McConnell M, Packard CJ, Shepherd J. Influence of plasma lipid and LDL-subfraction profile on the interaction between low density lipoprotein with human arterial wall proteoglycans. Atherosclerosis 1996; 124(2): 261-271 [PMID: 8830938]

15 Krauss RM. The tangled web of coronary risk factors. Am J Med 1991; 90(2A): 36S-41S [PMID: 1994714]

16 Austin MA, King MC, Vranizan KM, Krauss RM. Atherogenic lipoprotein phenotype. A proposed genetic marker for coronary heart disease risk. Circulation 1990; 82(2): 495506 [PMID: 2372896]

17 http://www.who.int/medicine

18 Jan MR, Nazli R, Shah J, Akhtar T. A study of lipoproteins in normal and pregnancy induced hypertensive women in tertiary care hospitals of the north west frontier province-Pakistan. Hypertens Pregnancy 2012; 31(2): 292-299 [PMID: $20860490 \quad$ DOI: $\underline{10.3109 / 10641955.2010 .507843]}$

19 Neboh, E.E., Emeh, J.K, Aniebue, U.U, Ikekpeazu, E.J., Maduka, I.C., Ezeugwu, F.O. Relationship between lipid and lipoprotein metabolism in trimesters of pregnancy in Nigerian women: Is pregnancy a risk factor? $\boldsymbol{J}$ Nat Sci Biol Med 2012; 3(1): 32-37 [PMID: 22690048 PMCID: PMC3361775 DOI: 10.4103/0976-9668.95944]

20 Udoh, A., Ndem, E., Itam, E., Odigwe, C., Archibong, E. Studies on Cholesterol profiles at various stages of Pregnancy. Niger Journal of Internal Medicine, 2002, 3, 26-34.

21 De, J., Mukhopadhyay, A., Saha, P.K. Study of serum lipid profile in pregnancy induced hypertension. Indian J Clin Biochem 2006; 21(2): 165-168 [PMID: 23105637 PMCID: PMC3453987 DOI: 10.1007/BF02912935]

22 Niroumand, S, Khajedaluee, M, KhademRezaiyan, M, Abrishami, M., Juya, M, Khodaee G, Dadgarmoghaddam. M. Atherogenic Index of Plasma (AIP): A marker of cardiovascular disease. Med J Islam Repub Iran 2015; 29: 240 [PMID: 26793631 PMCID: PMC4715400]

23 Flier, J.S. Biology of Obesity, In: Kasper,D.L., Fauci, A.S., Longo, D.L., Braunwald, E., Hauser,S.L., Jameson J.L., Eds. Harrison,s principles of Internal Medicine.17th Ed. NewYork, NY. McGraw-Hill, 2008, 462-464.

24 Kearns K, Dee A, Fitzgerald AP, Doherty E, Perry IJ. Chronic disease burden associated with overweight and obesity in Ireland: the effects of a small BMI reduction at population level. BMC Public Health 2014; 14: 143 [PMID: 24512151 DOI: 10.1186/1471-245814-143]

25 Dobiasova M, Frohlich J. The plasma parameter log (TG/HDL-C) as an atherogenic index: correlation with lipoprotein particle size 
and esterification rate in apoB-lipoproteindepleted plasma (FER(HDL)). Clin Biochem 2001; 34(7): 583-588 [PMID: 11738396]

26 Daniels LB, Laughlin GA, Sarno MJ, Bettencourt R, Wolfert RL, Barrett-Connor E. Lipoprotein-associated phospholipase A2 is an independent predictor of incident coronary heart disease in an apparently healthy older population: the Rancho Bernardo Study. J Am Coll Cardiol 2008; 51(9): 913-919 [PMID: 18308160 DOI: $10.1016 /$ j.jacc.2007.10.048]
27 Mudhaff, S.K. Atherogenic Index of plasma (AIP) AS a parameter in predicting Cardiovascular Risk in males compared to the conventional Dyslipidaemia Indices(Cholesterol Ratios). Karbala Journal of Medicine, 2013, 6(1), 1506-1513.

28 Kamel, M.A.,Mamoud, S.A., Elfetooh, H.A. In- Utero Exposure to Maternal Diabetes Increase the Risk of Vascular Diseases in the F1 Offspring in Rats. American Journal of Biomedical Sciences, 2014, 6(3), 201-216. 